\title{
Pour une phénoménologie de ce que la conscience néglige et que l'approche naturaliste détecte
}

Pierre Livet

\section{(2) OpenEdition}

1 Journals

Édition électronique

URL : https://journals.openedition.org/cps/444

DOI : $10.4000 / c p s .444$

ISSN : 2648-6334

Éditeur

Presses universitaires de Strasbourg

\section{Édition imprimée}

Date de publication : 30 septembre 2015

Pagination : 101-118

ISBN : 978-2-86820-917-7

ISSN : $1254-5740$

\section{Référence électronique}

Pierre Livet, « Pour une phénoménologie de ce que la conscience néglige et que l'approche naturaliste détecte », Les Cahiers philosophiques de Strasbourg [En ligne], 38 | 2015, mis en ligne le 03 décembre 2018, consulté le 10 novembre 2022. URL : http://journals.openedition.org/cps/444 ; DOI : https:// doi.org/10.4000/cps.444

\section{c) (i) (3)}

Creative Commons - Attribution - Pas d'Utilisation Commerciale - Partage dans les Mêmes Conditions 4.0 International - CC BY-NC-SA 4.0

https://creativecommons.org/licenses/by-nc-sa/4.0/ 


\title{
Pour une phénoménologie de ce que la conscience néglige et que l'approche naturaliste détecte
}

\author{
Pierre Livet
}

\section{Apparaître phénoménologique et naturalisation}

Le mot d'ordre de la phénoménologie est de décrire et analyser ce qui apparaît tel que cela apparaît. Il est sous-entendu que cela apparaît à la conscience. Or les approches naturalistes ne veulent pas s'en tenir, dans leurs analyses et expérimentations des activités cognitives, volitives et affectives, à ce qui apparaît à la conscience. Elles recherchent souvent à mettre en évidence des relations ou processus qui ne sont pas conscients. Il y a parfois un pont entre les deux: ces expérimentations peuvent nous faire prendre conscience de ce que nous n'avions pas conscience de certaines propriétés de notre activité cognitive. Par exemple, nous n'avons pas usuellement conscience des différences importantes de nos capacités de perception de notre champ central de vision, celui de la fovéa, et de notre champ périphérique. Nous ne pouvons lire qu'en fovéa, alors qu'en périphérie nous détectons très rapidement des mouvements, ou sommes plus sensibles à des lumières faibles. De cela nous n'avons pas de conscience immédiate, mais une fois avertis, nous pouvons devenir sensibles à la différence, et ne plus présupposer que nous pourrions lire en périphérie. Ou encore il pourra nous «sauter aux yeux" que le dessin d'Ernst Mach, où il représente tout ce qu'il peut embrasser de sa chambre d'un seul regard à partir de son fauteuil, ne correspond pas à ce qui nous apparaît perceptivement, puisque les éléments qu'il ne pouvait voir qu’en périphérie sont dessinés avec autant de détails que les éléments centraux. 
La naturalisation implique d'aborder nos conduites et expériences cognitives faites en première personne avec le point de vue de la troisième personne. Mais nous voyons sur l'exemple précédent qu'un résultat obtenu en troisième personne peut avoir des incidences sur une expérience en première personne. Cependant, on peut aussi obtenir des incidences de ce genre quand par un entretien bien orienté, donc dans une conversation entre première et seconde personne, on amène le sujet à découvrir des éléments et des détails dont il n’avait pas conscience qu'il les avait identifiés et mémorisés ${ }^{1}$. On peut d'ailleurs se demander si la résonance sur nos impressions conscientes des informations obtenues par une approche naturaliste ne passe pas aussi simplement par ce médium de la conversation - car on peut prétendre que la lecture en est une forme décalée. Mais le plus intriguant, c'est que, quelles que soient les sources de ces informations, il faut bien que le sujet ait le sentiment qu'il a la capacité, concernant ce qu'on lui révèle de ses processus cognitifs et qu'il n'avait pas aperçu, d'en tester la pertinence et la validité sur le mode de son expérience propre. Autrement dit, même si, pour que ces processus ainsi détectés apparaissent il faut souvent passer par des conversations avec autrui ou par des données externes au vécu, les effets de ces processus et parfois même ces processus eux-mêmes apparaissent bel et bien à la conscience subjective. Et cela implique qu'ils soient susceptibles d'une description et analyse phénoménologique.

Qu'en est-il des expérimentations en psychologie et en neurosciences qui mettent souvent entre parenthèses la manière dont le sujet perçoit les conditions d'expériences, ou qui montrent que les explications des résultats de ces perceptions ne correspondent pas à celles que notre conscience en première personne nous suggère? Par exemple, si nous avons à indiquer où s'arrête la trajectoire d'un mouvement d'un bras alors que nous n'en voyons que le début et que la fin de cette trajectoire est masquée, nous aurons tendance à penser que c'est notre seule anticipation visuelle, liée à la mémoire des représentations visuelles habituelles de ce genre de mouvement, qui nous permet de fixer ce point. Mais Saunier et al. ${ }^{2}$,

1 N. Depraz et al., "On Becoming Aware: The Pragmatic of Experiencing".

2 G. SAUnier et al., "Inference of complex human motion requires internal model of action: behavioral evidence", p. 399-409. 
ou encore Pozzo et al. ${ }^{3}$ ont montré, d'une part des différences de précision entre des trajectoires dont la dynamique était biologiquement et physiquement normale et d'autres dont la dynamique ne l'était pas, et d'autre part une surestimation de la longueur de la trajectoire cachée. Le deuxième effet s'explique si nos estimations dépendent de ce que l'œil qui suivait la trajectoire poursuit quelque peu sa course sur la lancée de son mouvement, et le premier si nous utilisons un savoir incarné des contraintes qui régissent ces dynamiques, dont le contenu est de type moteur et non visuel. Nous avons donc tendance à négliger l'influence de notre motricité dans nos anticipations perceptives. Pourtant, Husserl

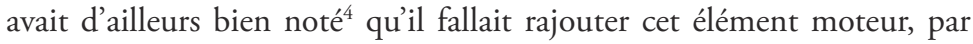
exemple pour comprendre l'enchaînement des phases de nos perspectives sur un objet autour duquel nous pourrions tourner, alors même que cet élément moteur n'est pas dominant dans notre conscience immédiate. L'avait-il fait par infraction au principe phénoménologique et par recours à ce qu'il savait, en géométrie et cinématique, de la continuité entre les perspectives? Ou bien son analyse de la perception lui donnait-elle les moyens de faire resurgir l'aspect moteur négligé? Une autre hypothèse est plus plausible: il aurait pris pour une évidence perceptive ce qui est plutôt un présupposé ou une croyance de base, la croyance que nos représentations perceptives vont avoir une continuité parallèle à celle de nos mouvements, alors qu'en fait les différentes facettes (les phases, justement) qu'un objet offre à notre perception quand nous tournons autour n'ont pas toutes la même pertinence perceptive - ainsi l'image que présente quelqu'un vu de dos, légèrement de côté, ne nous reste pas dans l'esprit.

Même si les expérimentations en neuropsychologie identifient souvent des processus qui sont trop rapides pour donner lieu en tant que tels à des images conscientes (vision subliminale, par exemple), et donc qui sont censés fonctionner "en aveugle", c'est-à-dire sans pouvoir être corrigés et réinterprétés en cours de route par une prise de conscience, on peut se demander s'il est vraiment possible d'expurger tout rapport de ces processus aux intentionnalités conscientes auxquelles ils sont sousjacents. En effet, s'ils peuvent intéresser un neurologiste ou un biologiste

3 T. Pozzo et al., "Kinematic features of movement tunes perception and action coupling", p. 75-82.

4 E. Hussert, Chose et Espace, Leçons de 1907. 
en tant que simples processus, quand ils en viennent à intéresser le psychologue ou le neuropsychologue, c'est bien par le rapport qu'ils ont avec des processus dont les résultats apparaissent à la conscience. Par exemple, l'analyse des saccades visuelles produites pendant une lecture, qui ne sont jamais des éléments de conscience immédiate et directe durant cette lecture, ne prend cependant d'intérêt que parce qu'elles sont des processus déterminants de la lecture, qui elle est consciente. Plus généralement, le neuropsychologue est amené à associer des repérages d'activités cérébrales avec des activités dont les différences tiennent à la manière dont elles apparaissent à la conscience, ou à ce que certaines sont conscientes et d'autres pas. Davantage, il ne peut se permettre de prétendre que des activités qui sont au premier plan de la conscience correspondent exactement aux mêmes activités cérébrales que celles des activités qui ne deviennent jamais conscientes, ni même que celles des activités qui sont accessibles à la conscience, mais qui ne parviennent au premier plan que par une conversion d'attention, ou que celles des activités qui ne pourront jamais faire l'objet que d'une conscience pour ainsi dire latérale. Autrement dit, il doit rendre compte des différences entre le mode de la conscience "transparente» - terme mal choisi par Metzinger pour désigner une conscience qui ne laisse pas apparaitre autre chose que son objet, et qu'il vaut mieux nommer une conscience "absorbée» - et un mode de conscience marginale de ce qui sert de fond à une forme perçue par la conscience absorbée, ou encore une conscience marginale de la présence possible d'éléments pertinents, mais dont on ne peut pas prendre de conscience claire. Il n'est pas jusqu'aux interprétations illusoires et aux fausses reconstructions rétrospectives qui ne doivent correspondre à des activités cérébrales différentes des perceptions directes. La naturalisation et la phénoménologie peuvent donc se relayer l'une l'autre.

\section{Cécité inattentionnelle}

Il est un domaine de phénomènes pour l'étude desquels ces entrelacements entre phénoménologie et naturalisation sont indispensables. C'est celui des marges de la conscience absorbée, ou

5 S. Dehaene et al., "Conscious, preconscious and subliminal processing: a testable taxonomy”, p. 204-2011. 
encore de ce que l'acte même de prise de conscience est amené à négliger. Nous avons déjà donné l'exemple des spécificités de la vision périphérique, voire de l'organisation des saccades visuelles (qu'on peut d'ailleurs apprendre à modifier quand on est dyslexique, ce qui donne une forme d'accès à ces processus qui ne sont normalement pas apparents). On peut encore mentionner la "cécité inattentionnelle», qui est paradoxalement un effet de l'attention, et qui tient à ce que lorsque nous nous focalisons sur un objet, une propriété de l'environnement ou encore une tâche de détection spécifique, nous devenons aveugles à d'autres éléments qui pourraient être pertinents, y compris parfois quand ils sont présentés au point de fixation de notre regard ${ }^{6}$. Nous sommes aussi aveugles au changement quand il est très progressif, ou qu'il concerne un élément d'arrière-plan non focalisé. Ou encore, l'attention portée à des mouvements ou des relations (par exemple suivre les mouvements d'un ballon que se relancent des joueurs) nous rend incapables d'identifier des éléments qui sinon nous apparaitraient saillants (le passage d'un comparse déguisé en gorille). Enfin, si dans une suite de présentations sur un écran on intercale rapidement un distracteur ou simplement un écran vide (attentional blink), cela peut nous faire négliger des changements, et même un changement d'élément pertinent pour la tâche en cours ${ }^{7}$.

Mais nous sommes aussi sensibles à des éléments qui ne mobilisent pas notre attention. Même dans ces expériences distractives, nous sommes capables en analysant nos souvenirs de retrouver des éléments que nous ne pensions pas avoir perçu. A tout le moins, nos réponses à des questions sur ces éléments seront meilleures que si elles étaient faites au hasard. Ainsi nous ne prêterons pas attention à la personne qui passe entre deux joueurs se relançant une balle, mais nous pourrons indiquer la couleur du ballon. Une autre modalité consiste à ressentir que "quelque chose ne va pas", que "nous n'avons pas tenu compte de quelque chose», qu' "il y a quelque chose d'incongru», que "quelque chose a changé», et cela sans savoir ce qui ne va pas, de quoi nous n'avons pas tenu compte,

6 D. Memmert, "The gap between inattentional blindness and attentional misdirection", p. 1097-1101.

7 A. Mack and J. Rock, Inattentional Blindness; D. J. Simons, C. F. Chabris, "Gorilla in our midst: sustained inattentional blindness for dynamic events", p. 1059-1074. 
ce qui a changé ou ce qui est incongru. Certes, nous avons aussi tendance à ne pas tenir compte de ces impressions dans nos décisions conscientes volontaires, mais cela n'empêche pas qu'un élément dont la présence peut perturber la réalisation d'une tâche ait d'autant plus d'influence perturbatrice qu'il ne donne pas lieu à identification consciente.

Tous ces résultats, qui ressortent du type "cécité inattentionnelle", sont obtenus par des protocoles d'expérience "naturalistes" qui ne mettent pas le sujet en position de "prendre conscience" de ce qu'il néglige pendant qu'il le néglige. Il faut bien cependant que le sujet puisse valider cette absence de prise de conscience. La manière "naturaliste» d'obtenir cette validation, c'est d'observer des défauts ou pannes dans la tâche entreprise de manière consciente. On a aussi recours à un mode de révélation similaire pour des "négligences", qui sont robustes en ce sens que même une fois avertis que dans les expériences qui les concernent nous allons négliger un changement (sans bien sûr désigner spécifiquement quel changement), nous ne parvenons pas à repérer ce que nous allons négliger. Cependant la surprise que nous ressentons une fois le changement révélé est bien une validation phénoménologique de l'existence de cette négligence.

\section{Les divers modes d'apparaître du négligé}

Mais ce n'est pas le seul mode phénoménologique d'apparaître du "négligé». Nous prenons ce terme non pas au sens le plus courant, celui de quelque tâche, propriété, forme ou objet dont nous avions une certaine conscience, mais sur lequel nous ne nous étions pas focalisés, si bien que nous n'avons pas accompli certaines activités qui lui étaient reliées, mais au sens de "ce que notre conscience, dans le cours de sa dynamique, oblitère ou laisse en marge de cette dynamique». Tentons d'explorer divers autres modes d'apparaître.

Dans la vie courante, hors protocole expérimental, nous pouvons aussi être avertis d'une négligence par une panne ou un défaut de notre tâche en cours, ce qui implique la conscience d'une rupture avec ce qui était implicitement anticipé dans le cours de cette tâche. Par exemple, si vous jouez d'un instrument comme l'orgue, où main gauche, main droite et pieds doivent suivre des parties de polyphonie différentes, vous pouvez entendre un désaccord harmonique, sans pouvoir repérer si cela vient de la main gauche, de la main droite ou des pieds. Quelque chose 
a été négligé, mais vous n’avez pas conscience de ce dont il s'agit. Il vous faudra parfois du temps pour le repérer, surtout si cela tient à ce qu'une ligne mélodique plus saillante a attiré une de vos mains ou vos pieds à en jouer une note, alors que le rôle qui leur était assigné devait être moins valorisé, puisque dans ce cas pour aucun des membres en collaboration vous ne recevez de signal que la note jouée ne figure pas sur la partition.

D'autres pannes sont immédiatement reconnues, ainsi quand vous avez le soleil presque dans les yeux et que vous ne voyez pas une voiture arriver d'une autre route parce qu'elle était dans l'ombre. Le négligé fait alors irruption dans votre perception, parce qu'il est en rupture avec les anticipations implicites ou explicites que comportait notre dynamique perceptive. Ces pannes et ruptures nous font prendre conscience qu'il y a eu négligence, mais sur le mode rétrospectif.

La cécité à un changement très progressif (qui peut nous rendre insensible à ce qu'une surface qui occupe $1 / 5$ d'un écran, par exemple le capot d'une voiture à l'avant-plan, change peu à peu du bleu au rose) donne aussi lieu à une découverte rétrospective de négligence, mais il faut cette fois qu'on nous signale le changement, nous n'arrivons pas à le découvrir tout seuls. Une fois informés, soit nous en restons à constater notre absence de repérage du changement, soit nous avons tendance à combler ce vide en nous remémorant, sans pouvoir être certains qu'il ne s'agit pas de faux souvenirs, quelques indices qui auraient pu nous le faire repérer. Cela peut même nous conduire à un redoublement de négligence, quand nous comblons ainsi des vides dans nos souvenirs en construisant des faux souvenirs qui restaurent une continuité psychologique, mais qui amènent à négliger cette fois l'absence de souvenir identifiable.

Il est intéressant de noter les similarités et différences de ce comblement interstitiel, source d'une négligence de second degré, avec la conscience interstitielle propre aux transitions d'une Gestalt à l'autre, quand nous percevons un dessin du genre lapin/canard, ou figure de vielle femme/jeune femme avec fourrure et chapeau, que nous mettons un peu de temps pour passer de l'une à l'autre, et que cette durée est consciemment perçue. Dans ce cas, nous ressentons pendant cette durée une résistance au changement de perspective, si bien que nous identifions un interstice durant lequel nous anticipons bien la Gestalt suivante, mais sans pouvoir la percevoir. L'interstice n'est pas un vide dans la conscience, mais découle de ce qu'une absence de perception se superpose à l'anticipation de la forme suivante. Une anticipation 
perceptive plus générale est ici aussi à l'œuvre: celle de n'avoir qu’à changer de perspective pour qu'aussitôt et sans délai changent les formes perçues. Cette anticipation est ici déçue, ce qui peut nous amener à nous douter qu'elle est source de négligences.

Une autre transition perceptive, celle qui nous mène de la focalisation sur une forme à la sensibilité à un élément de son fond, est plus rapide mais peut nous révéler aussi que nous venons de corriger une négligence, du moins quand cet élément ou ce détail est pertinent pour notre tâche en cours, voire active des motivations qui sont bien les nôtres, mais qui n'étaient pas l'instant d'avant mobilisées. Le rapport entre cet élément de fond et la tâche en cours peut aussi être plus conflictuel. Ainsi prendre une conscience focale d'un bruit de fond qui perturbait notre tâche nous fait prendre conscience à la fois de cette perturbation et de sa source ${ }^{8}$.

Dans ce genre de situation, nous identifions rétrospectivement un ressenti de malaise alors que notre focalisation sur notre tâche nous faisait le négliger. Or, dans bien des cas, nous ressentons que «quelque chose ne va pas" sans pouvoir identifier ce qui ne va pas, quel est le rapport avec la tâche en cours, si cela nécessite des ajustements utiles pour cette tâche ou si cela nous oriente vers d'autres activités. C'est là un mode de conscience qui, tant que nous restons focalisés sur la tâche en cours, nous fait prendre conscience du négligé tout en continuant à ne pas pouvoir nous focaliser sur lui, donc tout en le négligeant. Il rend effective une situation qui ne semblait pas possible dans une conceptualisation trop simple, où l'on confond conscience et conscience focale et absorbée, la focalisation sur le négligé étant impossible. Mais il est possible de faire l'expérience d'une conscience non focale, d'une forme d'aura de notre conscience focale - qui n'est pas pour autant une conscience "ouverte" comme dans la mindfulness, puisque la mindfulness prétend ne rien négliger, nous allons y revenir.

Il peut être intéressant de situer ces différentes formes de négligés par rapport à la typologie de Dehaene et al. ${ }^{9}$. Ces auteurs mettent en évidence des différences entre le subliminal non pris en compte (unattended, sans influence top-down), le subliminal pris en compte, le préconscient (qui

8 N. Depraz, dans Attention et vigilance, rapporte une expérience vécue où découvrir la source de la perturbation dissipe notre énervement, mais cela demande une attitude particulièrement «zen».

9 S. Dehaene et al., op. cit. 
pourrait être conscient s'il était renforcé par une influence top-down) et le conscient, qui implique ce renfort. Un changement très progressif est aussi de ce type. Les négligences qui se manifestent par des ruptures peuvent impliquer une transition du subliminal non pris en compte à l'un des trois autres états. Les pannes dont la source n'est pas encore identifiée impliquent cependant une prise en compte de la panne, et ressortent donc au moins du subliminal pris en compte. Les retards au basculement d'une Gestalt sur l'autre superposent une couche du mode subliminal pris en compte et une couche consciente. Le conflit entre le bruit de fond et la tâche aussi. Quand le fond, ou plus généralement un élément non focal, influence la focalisation de manière plus congruente, on peut penser à une relation entre préconscient et conscient.

Enfin, nous pouvons identifier quelques-unes de nos négligences par un retour réflexif qui arrive à différencier certains des divers aspects initialement entrelacés dans une de nos expériences conscientes. Mais pour que ce retour réflexif ne se réduise pas à la prise de conscience des pannes cognitives et à l'identification rétrospective de leurs sources, ou encore ne préside pas à des reconstructions illusoires, il faut que le flux de conscience n'ait pas été moniste et qu'il y ait eu au moins des indices de la diversité des courants composant ce flux. Et cela requiert des formes de conscience qu'on peut dire "accompagnantes», en ce qu'elles procèdent en compagnie de la conscience focale et absorbée, même quand elles donnent des indices du fourvoiement de cette conscience par exemple, quand nous avons fait un calcul que nous pensons juste, mais qu'un sentiment de doute continue à persister. Certes, toute forme de conscience «accompagnante» ne nous fait pas prendre conscience du négligé tout en le négligeant - certaines peuvent simplement alimenter notre focalisation, comme lorsque développer une idée en fait poindre une autre qui vient soit en renforcement, soit en développement, soit en contrepoint de la précédente. Mais, à l'inverse, toute conscience qui nous donne un accès marginal au négligé tout en ne déclenchant pas sa focalisation, donc tout en le négligeant, implique une forme d'accompagnement.

Cependant, les relations ne sont pas souvent iréniques entre ces différents courants de conscience. La focalisation et même la réflexion peuvent oblitérer des consciences accompagnantes au lieu de leur laisser place. La focalisation à elle seule produit de la négligence, puisqu'elle concentre les activités cognitives sur une donnée au détriment des autres. 
La prise de distance réflexive peut nous alerter sur cette négligence, mais elle implique forcément aussi quelque négligence, puisqu'elle se focalise sur une expérience consciente qui vient d'être en cours et qu'elle ne peut pas maintenir aussi dans sa focale la poursuite de cette conscience, ce qui prive le mode d'apparaitre phénoménologique de la conscience réflexive d'une des dimensions de la dynamique consciente. C'est seulement en combinant différentes "captures» d'expériences conscientes prises dans des étapes différentes de leur cours que la réflexion peut compenser cette négligence, mais alors elle est passée à une conscience qui synthétise diverses expériences, elle n'est plus un apparaître réflexif de «ce cours de conscience».

La conscience réfléchie ne peut donc compenser pleinement le déficit et la négligence indissociable de toute focalisation. Mais il est des formes de consciences moins focalisées, voire même qui prétendent à la nonfocalisation: les formes de conscience "ouverte». Celle qui a le moins de prétention est ce que les neurologues appellent le « mode du système par défaut", et qui correspond en principe à une forme de conscience flottante, qui ne s'attache durablement à rien de particulier (même s'il se trouve que ce système cérébral est aussi activé pour des informations qui nous touchent personnellement). Nous ne demeurons pas indéfiniment dans ce mode, et nous avons tendance à nous refocaliser, pour ensuite y revenir dans des moments de repos. Nous pouvons aussi nous exercer à sortir de telle ou telle focalisation. Des exercices de méditation, en particulier ce qu'on appelle la «mindfulness» ou pleine conscience, sont orientés en ce sens, et s'ils activent souvent cérébralement le mode par défaut, ils ne s'y réduisent pas. Est-ce qu'ils peuvent nous donner une conscience du négligé qui ne pollue pas son statut de négligé en nous focalisant sur lui?

La mindfulness implique de laisser flotter son esprit, en gardant un certain contrôle d'un aspect: quand un train de pensées commence à nous entraîner, il nous faut refuser de le suivre et l'abandonner, pour pouvoir maintenir la labilité de nos contenus de conscience. D'autres techniques de méditation commencent par nous focaliser sur une cible qui ne fait pas partie des objets normaux de focalisation. Il s'agit le plus souvent de la respiration, processus qui est perpétuel et en arrière-fond de toutes nos activités, si bien que nous n'avons pas normalement besoin de nous focaliser sur lui. D'autres rythmes corporels ne cessent jamais dans le cours de notre vie, comme le battement du cœur, sur lequel nous ne 
nous focalisons pas, du moins quand son intensité ne dépasse pas dans notre poitrine un certain seuil. Mais il est plus aisé de modifier à volonté le rythme de notre respiration que celui de notre cœur. Nous pouvons donc plus aisément nous focaliser sur l'entretien d'un rythme donné de respiration. Ce type de rythme a, du coup, la propriété de pouvoir nous absorber alors qu'il n'est pas du type focalisateur. C'est donc un parfait "défocalisateur", qui retient notre attention sans déclencher un train de pensée qui se développe, puisque le rythme qui revient n'ajoute rien d'autre qu'un nouveau cycle similaire. Certaines méditations impliquent de se concentrer sur un thème (souhaiter le bonheur des autres) mais, alors, c'est la répétition du thème, sans chercher à le développer de manière argumentée, qui assure la combinaison entre absorption et défocalisation.

Cependant, ces exercices, s'ils nous défocalisent et donc peuvent faire apparaître des processus négligés, ne nous orientent pas vers eux en tant que négligés, mais seulement parce qu'ils nous proposent de nouvelles accroches de concentration pour éviter les développements des focalisations.

Natalie Depraz, dans son livre Attention et vigilance, si riche en analyses phénoménologiques comme en références husserliennes, mais aussi psychologiques et neuropsychologiques, propose une notion d'attention ouverte, de vigilance ${ }^{10}$, qui diffère elle aussi de la conscience focalisée, mais qui n'implique pas nécessairement de passer par des défocalisateurs comme la respiration. Il s'agit d'une attention panoramique, ouverte, et affectivement réceptive. Elle permet aussi bien d'être réceptif aux autres et à leur altérité que d'être réceptif à l'expérience que nous vivons, en n'en écartant pas d'avance par focalisation certains aspects. Du coup, elle permet d'échapper à ce paradoxe qui tient à ce que l'attention est elle-même négligée, tout comme la respiration, parce qu'elle est sous ses différents aspects une dynamique quasi perpétuelle

10 Quand Dehaene et al. utilisent la notion de "vigilance», c'est, comme on l'a vu, pour désigner la conscience prise comme état, donc sans se soucier de sa relation transitive à un objet de conscience, et pour envisager des degrés d'activation de cet état, en particulier liés à des activations du thalamus, qui peuvent aller d'une activation minimale, donc du coma, à des activations plus intenses qui permettent une réceptivité, ce qui suppose alors des liens avec les activations de régions corticales. Ils n'envisagent donc pas l'attention ouverte que met en évidence Natalie Depraz. 
de notre existence consciente. Certes, notre conscience n'est pas toujours focalisée et absorbée par son objet, mais toutes nos expériences conscientes supposent une prise en compte des éléments pertinents pour nos motivations, même si la conscience focalisée peut négliger certaines prises en compte (ce que notent Dehaene et al.). L'attention ouverte, elle, nous permettrait de ne plus négliger l'attention. Mais on voit que cela ne peut devenir possible que si nous n'obéissons pas à la dominance de l'attraction de l'attention focalisée.

Assurément, l'ouverture à l'attention comme processus négligé, en tant qu'ubiquiste, pourrait bien être une manière de reconnaître son statut de processus négligé, comme tel. Mais l'attention ouverte ne permet pas nécessairement d'analyser plus précisément les processus qui font du négligé un élément indispensable à toute conscience, et qui permettent d'amorcer la sensibilité à ce négligé au cœur même de nos focalisations. En effet, l'attention ouverte se donne son ouverture par principe, comme une dynamique de base, elle ne nous dit pas comment cette ouverture subsiste dans les interstices des focalisations et comment elle les alimente, ce qui est pourtant le statut du négligé (statut qui n'était pas, il faut le mentionner, l'objet du livre de Depraz).

\section{La conscience liquide}

L'attention ouverte, cependant, fait bien partie des modalités de conscience qui n'oblitèrent pas le négligé, et qui pourraient donc le laisser apparaître. Tentons de déterminer quelques unes de ces modalités.

Le mode par défaut ou la conscience flottante de celui qui rêvasse n'oblitèrent pas le négligé, mais ils ne favorisent pas spécialement son apparaître, qui en partant d'eux peut se produire de façon seulement aléatoire, de manière plus ou moins équiprobable avec l'apparaitre d'autres modes de conscience. L'attention ouverte, elle, favorise une sensibilité aux différentes qualités des divers modes de conscience. Elle peut donc nous rendre sensible à la différence entre un mode focalisé et les différents modes d'apparaitre du négligé que nous avons recensés: surprise, une fois un changement révélé alors qu'on ne l'avait pas vu venir, panne et perturbation de l'activité cognitive en cours, discontinuité ou retard d'un processus cognitif par rapport au rythme anticipé (par exemple entre deux Gestalten), transition d'une perception d'une forme à une perception d'un élément de son fond, conscience accompagnante 
qui suggère que nous avons manqué une information, ou que nous pourrions suivre une autre piste cognitive, attention réflexive qui met l'accent sur différents plans de conscience dans une même expérience.

Cette attention réflexive différenciatrice utilise le fait que toute conscience apparait sur le fond de processus accompagnants, dont on pourrait comparer la relation qu'ils ont avec la dynamique consciente focalisante à celle qu'ont en dynamique des fluides, avec le mouvement d'un poisson, les déplacements de liquides qui l'accompagnent. Dans ces dynamiques, se forme une couche laminaire proximale, qui est au contact du corps et est quasi immobile par rapport à lui (au sens où elle se meut avec lui), puis on note des couches turbulentes, et c'est au delà de ces couches qu'on arrive aux flux d'écoulements réguliers du liquide ambiant. Cette analogie nous rappelle qu'entre ce qui est ignoré (le courant régulier du fluide), et ce qui absorbe la conscience focalisée (qui a le rôle du mouvement du poisson dans l'analogie), on trouve ce qui est négligé et qui pourrait nous orienter sur des pistes différentes - en lui associant les couches turbulentes - mais aussi la différence propre à la modalité de conscience en cours, en particulier la négligence de l'attention à elle-même, dont le statut est en un sens similaire à celui de la couche laminaire. Quand nous sommes pris par une attention focalisante, cette modalité manifeste sa différence avec d'autres modes possibles, non pas en évoquant ces autres modes et en s'y comparant, mais simplement en ayant sa tonalité propre: l'attention porte avec elle une tonalité de concentration. Cette modalité en tant que contenu conscient est "négligée», au sens où elle ne fait évidemment pas partie de ce sur quoi nous nous focalisons, mais il s'agit d'une négligence différente de celle que nous avons associée à ces analogues des turbulences que sont des flux de conscience marginaux ou transverses. C'est une négligence qu'il est plus pertinent d'associer à cette couche laminaire proximale, qui n'est pas en mouvement par rapport à la nage du poisson, mais qui suit exactement le déplacement de son corps.

La réflexion phénoménologique ne serait pas possible sans cette négligence, qui consiste en fait non pas à laisser de côté quelque chose, mais au contraire à ce qu'une certaine forme de négligé ne «décolle» pas de l'activité en cours. C'est ainsi que pouvons avoir le sentiment d'être engagé dans "une» modalité de conscience et pas dans une autre. Ce sentiment d'identité n'est pas assuré par une identification de la modalité qui serait interne à l'activité en cours, puisque cela demanderait une prise 
de distance qui nous ferait passer dans une autre modalité. Mais il peut être assuré simplement par l'absence d'un décollement qui signalerait la présence d'un autre type d'activité. Plus généralement, notre manière de nous trouver en unité avec nous-mêmes (ou avec le monde) ne tient pas à ce que nous nous focalisons sur cette unité - elle serait au contraire compromise par cette autre focalisation - mais simplement à ce que nous n'avons aucun indice de décollement.

Comme nous avons pu assigner un type phénoménologique, une forme d'identité, aux autres formes de négligés, ceux que nous associons ici aux turbulences, et qui peuvent d'ailleurs, comme elles dans leur rapport au mouvement du poisson, soit perturber l'activité soit au contraire l'aider, nous pouvons supposer qu'au moins certaines formes d'apparaître à la conscience du négligé (turbulent) comme tel doivent aussi impliquer un mode de non décollement, une couche laminaire qui envelopperait aussi les tourbillons. Il s'agit bien alors de ce que nous avions visé en parlant d'un apparaître du négligé «en tant que tel ». Notre analogie permet ici de comprendre que pour saisir le négligé comme tel, nous n'avons pas besoin de prendre de la distance par rapport à lui et en même temps de maintenir le fait que notre conscience le néglige. Il nous suffit de ne pas avoir d'indice de décollement avec cette situation de négligence. Certes, une différence persiste avec l'absence de décollement qui indique une unité simple de la conscience subjective: l'absence de décollement avec la, ou les négligences présuppose l'admission d'une diversité simultanée de modes de conscience, qui se développent en quelque sorte en filigrane soit par rapport au mode d'attention focalisé, soit les uns par rapport aux autres, par exemple dans l'attention ouverte. Mais elle présuppose aussi que ces modes simultanés sont entrelacés, puisque sinon, nous aurions des indices de décollement.

La possibilité de ressentir, alors même qu'on est focalisé sur un contenu, une différence entre le mode focalisé et un négligé non focalisé qui lui est marginal, et cela sans avoir à se focaliser sur ce négligé, exige de renoncer à une conception simple et symétrique de la négation. Quand nous utilisons la négation classique, non (non $\mathrm{A})=\mathrm{A}$. Dans cette version simplifiée, être inattentif (non A) est la négation de l'attention focalisée (A). Donc être attentif à être inattentif est la négation de cette négation (non(non A)), et c'est par conséquent une attention focalisée (A). Si on prétend que ce n'est pas cela, alors on affirme non(non(non A), et cela reviendra à non $\mathrm{A}$ : être inattentif. Mais ne pas ressentir de décollement 
n’a pas la même portée que cette double négation (dite involutive), qui reviendrait alors à une identité simple. Cela revient seulement à ne pas nous donner de raison suffisante de penser que nous sommes vraiment multiples et sans unité. Et cela permet de prétendre à une forme d'unité sans avoir à nier l'existence d'une diversité de modalités d'impressions et de conscience. Inversement, cela permet de ressentir des indices de diversité de ces modes sans pour autant devoir isoler celui dont le mode de présentation est celui d'un apparaitre en filigrane, qui par définition n'apparaît jamais en isolation.

Quand on se demande si ces modes d'apparaitre en filigrane de nos négligences sont des informations ou impressions métacognitives, ou simplement para-, ou péri-cognitives, on peut raisonner de manière similaire. Si le méta-cognitif implique une prise de distance surplombante, une forme d'enchâssement dans laquelle ce qui est «méta-» ne figure que hors de la parenthèse (Méta $(\mathrm{Cog})$ ) et que ce dont il est le «méta-» ne figure que dans l'intérieur de la parenthèse, alors nous ne pouvons pas voir apparaître le négligé sur ce mode méta-cognitif. Mais si l'accompagnement d'une conscience focale par son négligé fait partie de ce qui permet la focalisation, et en même temps assure le non décollement entre conscience focale et négligé, alors ce méta-cognitif va simplement suivre les relations de différences et d'entrelacement entre les deux modes, et va ainsi donner des éléments pour analyser les influences mutuelles du focal et du négligé, et les incidences de ces négligences.

\section{Conclusion}

Nous devons donc développer pleinement les implications du renoncement à l'idée simpliste, selon laquelle le paradigme de la conscience orientée vers son objet est la conscience "transparente" à cet objet - au sens où elle est une conscience absorbée, qui ne donne accès qu'au contenu visé de l'objet et pas à ses propres modalités. Il faut alors distinguer «transparence» et "adhérence». Il y a transparence quand la conscience est absorbée par son objet. Mais il peut y avoir "adhérence», donc concentration sur un objet, sans qu'il y ait absorption et donc transparence. Dès lors, il est possible d'être orienté sur un objet de conscience tout en étant sensible aux modalités de conscience en filigrane, et donc au négligé. Il est donc possible d'être orienté sur les indices de négligences sans être absorbé par ces indices. 
Le mode d'apparaître de la conscience à elle-même ne semble donc pas avoir la structure de couches superposées distinctes et sédimentées, ou de rayons d'orientations différentes, mais plutôt ressembler à un fluide mu selon divers courants. Chacun de ces courants, ou registres de la conscience, peut être plus ou moins absorbant, mais aucun ne peut se séparer des autres. Les dynamiques conscientes sont celles d'un fluide multi-flux. Sous les flux dominants peuvent apparaittre en filigrane d'autres flux, soit laminaires, soit en forme de remous et turbulences. Ces turbulences peuvent se manifester seulement par des perturbations du flux dominant, ou elles peuvent s'entrelacer avec ce flux en créant en lui des divergences. Nous pouvons alors prendre une conscience latérale de ces divergences sans avoir à les travestir en flux dominants. La conscience focalisante, elle-même, retire sa phénoménalité de ce qu'elle inhibe au moins partiellement l'expression des autres flux, qui ont cependant toujours une influence sur cette conscience. Le mode d'apparaître de la spécificité de cette conscience focalisante prend appui sur ce rapport d'inhibition partielle. Pour analyser la spécificité d'un mode de conscience, y compris celui de la conscience focalisante, il faut donc utiliser ce mode même dans sa différence, donc, paradoxalement, procéder de la même manière que lorsque nous utilisons les modalités en filigrane. L'apparaître phénoménologique ne serait pas possible sans ces divers courants entrelacés dans un flux qui, sans nous certifier son unité, met hors jeu la possibilité d'un décollement mutuel des courants de conscience (mais ne le rend pas impossible, par exemple dans la schizophrénie). On pourrait soutenir que la conscience s'apparaît à elle-même parce qu'elle est auto-relationnelle. Mais en fait, elle est auto-différentielle, et le préfixe "auto" ne peut pas prétendre désigner une auto-référence garantie: cette connivence avec soi est seulement soutenable par défaut, par absence de décollement.

C'est sans doute sous cette modalité qu'il faut entendre la deuxième partie du mot d'ordre phénoménologique «décrire ce qui apparaît... tel que cela apparaît", qu'on pourrait renforcer par: «seulement comme cela apparaît», où le "seulement» n'est pas une purification, mais la reconnaissance d'une limitation. Comme l'approche par naturalisation tente de préciser les différences entre les diverses modalités de conscience, et plus largement les relations de la conscience avec les divers courants cognitifs, pas forcément conscients, qui l'alimentent, les deux approches non seulement sont compatibles, mais encore se nourrissent l'une l'autre. 


\section{Bibliographie}

Bishop S.R. et al., "Mindfulness; A proposed operational definition", Clinical Psychology: Science and practice, Autumn 2004, 11, 3, p. 230-241.

Dehaene S., Changeux J.-P., Naccache L., Sakur J., Sergent C., "Conscious, preconscious and subliminal processing: a testable taxonomy", Trends in Cognitive Sciences, vol. 10, n 5, May 2006, p. 204-2011.

Depraz N., Attention et vigilance; À la croisée de la phénoménologie et des sciences cognitives, Paris: PUF, 2014.

Depraz N., Varela F.J., Vermersch P., "On Becoming Aware: The Pragmatic of Experiencing”, Amsterdam: Benjamin Press, 2003.

Hasenkamp W., Wilson-Mendenhall Ch. D., Duncan E., Barsalou L., "Mind wandering and attention during focused meditation; a fine-grained temporal analysis of fluctuating cognitive states", Neuroimage, vol. 59, (1), 2012, p. 750-760.

Husserl E., Chose et Espace, Leçons de 1907, trad. J.-F. Lavigne, Paris: PUF, 1989.

Mack A., Rock J., Inattentional Blindness, Cambridge MA: MIT Press, 1998.

Memmert D., "The gap between inattentional blindness and attentional misdirection", Consciousness and Cognition, 19, 2010, p. 1097-1101.

Pozzo T., Papaxanthis Ch., Petit J.-L., Schweighofer N., Stucchi N., "Kinematic features of movement tunes perception and action coupling", Behavioural Brain Resarch, 169, 2006, p. 75-82.

Saunier G., Papaxanthis Ch., Vargas C.D., Pozzo T., "Inference of complex human motion requires internal model of action: behavioral evidence", Exp Brain Res, 185, 2008, p. 399-409.

Simons D.J., Chabris C.F., "Gorilla in our midst: sustained inattentional blindess for dynamic events", Perception, vol. 28, 1999, p. 1059-1074.

Thirioux B., Mercier M. R., Blanke O., Berthoz A., "The cognitive and neural time course of empathy and sympathy: an electrical neuroimaging study on self-other interaction", Neuroscience, 267, 2014, p. 286-306. 